\title{
THE FIFTY-FIRST REGULAR MEETING OF THE SAN FRANCISCO SECTION
}

The fifty-first regular meeting of the San Francisco Section of the Society was held at Stanford University on Saturday, April 2, 1927. The Chairman, Professor Allardice, presided. The total attendance was 45 , including the following 29 members of the Society:

Allardice, Barter, Bernstein, Blichfeldt, Buck, Cairns, Cajori, Collier, Corbin, Eells, R. L. Green, M. W. Haskell, E. R. Hedrick, Hoskins, Hotelling, Glenn James, Vern James, D. H. Lehmer, D. N. Lehmer, S. H. Levy, W. A. Manning, Moreno, Neikirk, Noble, T. M. Putnam, Pauline Sperry, Stager, A. R. Williams, Wong.

It was decided to hold the next Spring meeting at Stanford University on Saturday, April 7, 1928.

Titles and abstracts of papers read at the meeting follow. Professor Bell's paper was read by title.

1. Dr. J. D. Barter: On differential p-vectors.

Those of the fundamental properties of differential $p$-vectors which appear pertinent to the theory of partial differential equations and of integral invariants are developed. Since a differential is characterized by the algebraic invariants of itself and of its vector derivative, considerable attention is given to these. Exception is taken to certain theorems and proofs of Gegenbauer, Schouten, and others, and corrections suggested. The nature of the automorphic transformations of differential $p$-vectors is specified and consequences indicated.

2. Dr. J. D. Barter: Powers of determinants.

It has seemed desirable to place on record certain determinant forms which may be given to the powers of determinants since the direct proof of the relations in question appears difficult and useful applications seem probable.

3. Dr. J. D. Barter: On covariant differentiation. Preliminary communication.

The two types of covariant differentiation, termed respectively albraic and vector, are derived and their mutual relation developed. Certain apparently novel results are obtained and applied to the theory of Jacobi's last multiplier and its generalization.

4. Professor E. T. Bell: The arithmetic of logic.

It is shown that the algebra $L$ of logic is abstractly identical with certain parts of the arithmetic $A$ of the rational integers. Formal equiva- 
lents in $L$ of the G.C.D., L.C.M., congruences, primes, arithmetical divisibility and the unique factorization law in $A$ are developed. Lasker's concept of the residual in modular systems is necessary to complete the theory of congruences in $L$; it gives the equivalent in $L$ of dividing (when possible) both members of a congruence in $A$ by a common factor. There are two abstractly identical arithmetics of $L$, corresponding to the PeirceSchröder dualism in $L$. The theory has applications to the classification of "doctrinal functions" (Keyser), or "system functions" (Sheffer). This paper will appear in the Transactions of this Society.

5. Professor W. D. Cairns: Development in a system of approximately orthogonal functions.

As is well known the terms of the expansion of $(1+1)^{2 n}$, multiplied by a conveniently chosen factor, approach the ordinates of the curve $y=e^{-x^{2} / 2}$ as $n$ becomes infinite. This series of terms, and the principal parts of the successive differences formed therefrom, give polynomial functions which are dealt with in this paper. When combined by a summation analogous to the integration of orthogonal functions, these functions are shown to be orthogonal to within an error involving terms in $1 / n$. A development of suitably restricted functions in terms of these functions is given, the approximation holding to within the order $1 / n$.

6. Professor Florian Cajori: The earliest arithmetic published in America.

The author gives a description of the arithmetic of Pedro de Paz, a copy of which (partly in the original and partly in photostat reproduction) has been secured by the Library of the University of California. It was published in the city of Mexico in 1623. It is the earliest volume, on arithmetic exclusively, printed in America.

7. Professor Florian Cajori: Unpublished letters of C. $H$. Schumacher and W. Struve to F. R. Hassler.

The author gives an account of letters written by C. H. Schumacher and W. Struve to F. R. Hassler relating to astronomical publications, the design of improved geodetic instruments, and the procuring from Europe of expert copper engravers.

\section{Professor Florian Cajori: The logarithms of Napier.}

The author, with the aid of quotations from Napier, points out the invalidity of the argument against the accuracy of the formula Nap. $\log x=10^{7} \log \left(10^{7} / x\right)$, given on page 538 of the Proceedings of the National Academy of Sciences (September, 1926).

9. Professor E. R. Hedrick: On certain expansive properties of neighborhoods in general spaces.

Let us define as an expansive property of a region any property that holds true in any region provided it holds true in some region interior to the first one. Blumberg has recently presented a paper to the Society 
(Philadelphia, Dec. 29, 1926) regarding extensions of the theorem that any derived set is closed. If a set is defined in any space that has the enclosable property, by means of an expansive property of the enclosing regions about each point of the set, it is shown in the present paper that the set is closed. Examples are cited, and applications to the theory, particularly to the validity of a Borel theorem, are mentioned.

10. Dr. Harold Hotelling: Differential equations subject to error.

A generalization of the theory of differential equations is obtained if we associate with every point, instead of a fixed direction, a frequency distribution of directions. In this way a frequency distribution can be be found for curves. It is argued in this paper that when an empirical law has been derived from a differential equation and statistical verification is sought, a more accurate test may often be applied to the differential equation itself than to its integral. The constants in the differential equation are then best determined first, the constants of integration afterward. These considerations are applied to the "logistic" hypothesis and the numerical work carried out for the population of the United States. Intercensal interpolation becomes a problem in the calculus of variations. For estimates by means of this theory the determination of probable errors is connected with the theories of random migration and heat conduction.

11. Professor Glenn James: A theorem on monotonic decreasing functions.

In evaluating certain monotonic decreasing series, need for further classification of such series arises. Toward meeting this need we formulate and prove the following theorem. If $\phi_{0}(x)$ is positive and possesses derivatives to the nth order, those of even order being positive or zero, and those of odd order being negative or zero, and $\phi_{i}(x)$ denotes $\phi_{i-1}(x)-\int_{x}^{x+b} \phi_{i-1}(z) d z$, $1 \geqq b \geqq 0$, then the same rule of signs applies to $\phi_{i-1}(x)(i=1,2,3, \cdots, n)$ and its first $n-i$ derivatives. The theorem can be extended to functions of several variables.

12. Mr. D. H. Lehmer: Note on the largest Mersenne number.

The remarkable accuracy with which the famous statement of Mersenne in 1644 represents the facts concerning the primality of $2^{n}-1$ is well known. The present author finds however that the largest number, $2^{257}-1$, given by Mersenne as a prime is really composite. This fact is revealed by the application of Lucas' test to the number. It was found that the 256 th term of the recurring series $4,14,194$, is not divisible by $2^{257}-1$. It follows then this 78 -digit number is composite although it is impossible to say what its factors are. The test was undertaken in order to verify a similar calculation made by M. Kraitchik leading to the same general result. It has not been possible as yet to compare the two calculations. 
13. Mr. D. H. Lehmer: On the converse of Fermat's theorem.

This paper appears in full in the present issue of this Bulletin.

14. Professor L. I. Neikirk: A class of continuous curves defined by motion which has no tangent lines.

The first example of a continuous curve without tangents was given by Weierstrass in 1872. Since then several others have been given. The most notable are those given by Peano, by Hilbert, by E. H. Moore, (On certain crinkly curves), and by W. F. Osgood, ( $A$ Jordan curve of positive area). The novel feature of the curve here discussed is its definition by linear and rotational motion which makes the lack of tangents geometrically evident. The paper includes an analytic proof of this fact.

15. Dr. A. R. Williams: The quintic surface with two double straight lines.

The quintic surface with two double straight lines is obviously universal. The plane system is composed of quintics having in common two double points and twelve simple points. There must be a relation between these fourteen points; for if taken arbitrarily they would impose eighteen conditions on the quintics. It is shown that they are the base points of a pencil of quartics that have a common tangent at the two which are the double points of the quintics. The class of the surface is in general thirty-four. There are twelve pinch points, six on each double line. There are, in general, thirteen lines which meet both double lines. Certain special cases are noted, wherein nodes or additional lines are produced by particular positions of the base points.

16. Dr. B. C. Wong: Sextic surfaces with a double septic curve.

By an involutorial quartic transformation by means of four hyperquadric surfaces in 4-space a plane $\sigma$ is transformed into a two-dimensional variety $\sum_{2}^{6}$ of order 6 . Projecting $\sum_{2}^{6}$ from any point $z$ in $S_{4}$ on to a $S_{8}$ we obtain a surface of order 6 with a double septic curve. By taking $z$ in positions related to $\sum_{2}^{6}$ or by having the base points, 10 in number, in $\sigma$ take special relative positions, we obtain numerous special cases of the surface. This paper will appear in the Annals of Mathematics.

\author{
B. A. Bernstein, \\ Secretary of the Section.
}

